\title{
Book review: Gute Sorge ohne gute Arbeit? Live-in-Care in Deutschland, Österreich und der Schweiz
}

\author{
Karin Pape ${ }^{1,2}$ \\ ${ }^{1}$ Women in Informal Employment: Globalizing and Organizing (WIEGO), Manchester, UK \\ ${ }^{2}$ International Domestic Workers Federation (IDWF), Hong Kong \\ Correspondence: Karin Pape (karin.pape@wiego.org)
}

Published: 30 November 2021

Aulenbacher, B., Lutz, H., and Schwiter, K. (Hrsg.): Gute Sorge ohne gute Arbeit? Live-in Care in Deutschland, Österreich und der Schweiz, Beltz Juventa, Weinheim, 264 S., ISBN: 978-3-7799-6260-1, EUR 24,95, 2021.

Gute Sorge - ohne gut Arbeit? Mit dieser Frage greifen die Autorinnen das Kernproblem häuslicher 24-Stunden-Pflege auf. Wie fair kann ein Modell sein, das hohe Einkommensunterschiede zwischen Pflegebedürftigen und Pflegenden als Funktionsvoraussetzung hat?

Dass der Arbeitsplatz Privathaushalt ein besonderer ist, ist spätestens seit der Verabschiedung des ILOÜbereinkommens 189 ,Übereinkommen über menschenwürdige Arbeit für Hausangestellte“ (2011) allgemein anerkannt und global definiert. Die Definition der Arbeit geschieht über den Arbeitsort und die berufsmäßige Verrichtung (also gegen Bezahlung) und nicht darüber, welche spezifischen Arbeiten im Privathaushalt verrichtet werden.

Im europäischen Kontext wird grob zwischen ,putzen“ und „pflegen“ unterschieden, wobei die Grenzen fließend sein können. Die Kategorie der Live-in-Betreuerinnen in Europa ist eine spezielle, nicht nur wegen der besonderen Arbeitsbedingungen (Rund-um-die-Uhr-Verfügbarkeit, wohnen im Haushalt der zu betreuenden Person), sondern auch weil hier wohlfahrtsstaatliche und grenzübergreifende Regime berührt sind - jenseits eines privaten Arbeitsverhältnisses. Von den untersuchten Ländern - Deutschland, die Schweiz und Österreich - haben sowohl Deutschland als auch die Schweiz das ILO-Übereinkommen 189 ratifiziert und sich damit verpflichtet, die globale Regelung in nationales Recht umzusetzen. Der Grundgedanke der Konvention ist die Gleichbehandlung der Hausangestellten mit anderen Arbeitnehmer:innen allgemein in einem Land unter besonderer Berücksichtigung des Arbeitsortes (explizit im Artikel 6).
Die Autorinnen machen schon im Titel deutlich, worum es ihnen geht: Im Vordergrund steht die „gute Arbeit“ dieser speziellen Form der Pflegeerbringung - eingebettet in die verschiedenen Arrangements der drei untersuchten Länder Deutschland, Österreich und der Schweiz. In allen drei Ländern basiert das Modell einer Live-in-Pflegkraft auf der angeblichen Win-win-Situation der transnationalen Wohlfahrtsgefälle, die dazu führen, dass im Wesentlichen osteuropäische Migrantinnen in deutschen, schweizerischen und österreichischen Privathaushalten rund um die Uhr Pflegebedürftige versorgen und dafür zwar keine national adäquate Vergütung bekommen, aber doch viel mehr verdienen als in ihren Herkunftsländern.

Kann der Wunsch, im Alter gut betreut zu Hause zu leben, mit einer 24-Stunden-Betreuung zu fairen Bedingungen überhaupt gewährleistet werden? Obgleich die Autorinnen schon zu Anfang eine Antwort geben, indem sie feststellen, dass bei der 24-Stunden-Betreuungsform ,... gute Sor$g e$ versprochen und auch erwartet wird, ohne dass dabei $g u$ te Arbeit möglich wird“" (S. 10, Hervorhebungen durch die Autorinnen), durchzieht diese Grundfrage das Buch wie ein roter Faden.

Im 1. Teil werden interessierte Leser:innen durch die $\mathrm{Ge}-$ mengelage verschiedener rechtlicher Arrangements geführt (sowohl in den jeweiligen Ländern als auch im Vergleich der drei untersuchten Länder), einschließlich der Rechte auf dem Papier und in der Praxis. Dabei wird immer der Grundsatz im Auge behalten, dass Arbeitsrechte dazu da sind, bestehende Ungleichheiten und damit Machtkonstellationen auszubalancieren. Unter dieser Prämisse ergibt sich logisch ein kritischer Blick auf jegliche Form eines Selbständigkeitsmodells, das besonders in Österreich dominant ist. Selbständigkeit ist die Zauberformel, sich - völlig legal - jeglicher Verantwortung für die Anwendung von Arbeitsschutzgesetzen 
zu entziehen und damit alle Risiken, einschließlich Vorsorge im Krankheitsfall oder für das Alter, komplett auf die Beschäftigten abzuwälzen. Das gilt auch für Selbstständigenmodelle in der häuslichen Pflege, die oftmals in der Praxis wie Angestelltenverhältnisse gehandhabt werden.

Die zwingende Anstellung in einem Angestelltenverhältnis ist allerdings noch lange kein Garant für ,gute Arbeit", wie im Schweizer Beispiel beschrieben wird (Kapitel 4). Trotz sektoraler Mindestlöhne fehlen z.B. verbindliche Bestimmungen zu den Arbeitszeiten, und damit bleibt die Wirkung der Mindestlöhne beschränkt (S. 80), da generell der Arbeitsplatz Privathaushalt vom Schweizer Arbeitsgesetz ausgeschlossen ist.

Eine Tabelle (S. 24) gibt gleich zu Beginn einen guten Überblick über die Beschäftigungsformen, Anzahl der Betreuer:innen und Agenturen, wichtige Herkunftsländer, sozialstaatliche Einbettung und Absicherung, arbeitsrechtliche Absicherung und Interessenvertretung der drei untersuchten Länder.

Der 2. Teil beschäftigt sich intensiv mit der Besonderheit der Arbeit einer Live-in-Pflegekraft aus den verschiedenen Perspektiven der Agenturen, Familien der Pflegebedürftigen und der Live-in-Betreuerinnen. Zitate aus den geführten Interviews illustrieren eindrücklich die Problemlagen und die Ansätze der verschiedenen Akteur:innen. Auf ein generelles „Agentur-Bashing“ wird erfreulicherweise verzichtet. Es wird allerdings sowohl die prekäre Situation der Pflegebedürftigen als auch der Pflegekräfte in den Blick genommen. Die Rolle der Familien der zu pflegenden Personen wird als zentral formuliert, da sie diejenigen sind, die die Anforderungen von außen formulieren und sich oft gleichgültig oder ignorant gegenüber den Arbeitsbedingungen der Pflegekräfte verhalten (S. $160 \mathrm{ff})$. Auch hier wird deutlich, dass der gesellschaftliche Diskurs sich im Wesentlichen um die ,gute Sorge" und nicht so sehr um ,gute Arbeit" sorgt. Wer weiß schon - oder will wissen - wie der Alltag einer Live-in-Betreuerin aussieht? Und wie regelt sie ihre Abwesenheit zu Hause in ihrem Herkunftsland, wo nun auch eine Versorgungslücke entstanden ist?

Teil 3 wendet sich der Frage zu, welche Ansatzpunkte und Perspektiven es für gute Sorge und gute Arbeit gibt. Hier wird als Erstes auf die Selbstorganisation der Betreuer:innen in der Schweiz eingegangen - ein Thema, was in vielen anderen Veröffentlichungen nicht vorkommt. Die erfolgreiche Strategie, durch gewerkschaftlich unterstütze Gerichtsverfahren entscheidenden Einfluss auf die Verbesserung der Arbeitsbedingungen zu nehmen, ist auch für andere Länder anwendbar ${ }^{1}$. Die Darstellung der Widerständigkeit der Be-

\footnotetext{
${ }^{1}$ Das deutsche Bundesarbeitsgericht hat jüngst in einem Urteil (https://www.bundesarbeitsgericht.de/presse/gesetzlichermindestlohn-fuer-entsandte-auslaendische-, betreuungskraefte-inprivathaushalten/, letzter Zugriff: 28. November 2021) entschieden, dass die Rund-um-die-Uhr-Bereitschaftszeit einer bulgarischen Pflegekraft mit dem in Deutschland gültigen Mindestlohn zu
}

treuer:innen hebt sich wohltuend von vielen Beschreibungen in anderen Veröffentlichungen und Medienberichten ab, die den Betreuer:innen nur eine Opferperspektive erlauben. Die Beschreibung der erfolgreichen Selbstorganisierung der vermeintlich „Unorganisierbaren“ verdeutlicht den essentiellen Beitrag der eigenen Interessenvertretung als Teil jedweder Lösung.

Des Weiteren werden verschiedene Ansätze fairer Live-inBetreuungen diskutiert. Interessant ist hier u. a. der Ansatz der seriöseren Agenturen (Kapitel 11), die auf Qualitätsstandards und Zertifizierung setzen, um ,... sich damit Zugang zu den Leistungen der Pflegeversicherung verschaffen (wollen) (S. 197)“. Diese Agenturen setzen auf Legalität und damit auf Rahmenbedingungen, die es ihnen erlauben, sich von anderen Agenturen zu distanzieren, die im Graubereich unklarer oder fehlender rechtlicher Grundlagen operieren.

Einmal mehr stellt sich die Frage, ob ein Modell, das auf grenzüberschreitender Ungleichheit aufgebaut ist und die allzeitige Verfügbarkeit von Betreuer:innen verlangt, überhaupt fair sein kann. In diesem Zusammenhang werden Ansätze diskutiert, die andere Formen, wie z. B. Wohngemeinschaften oder auch gemeinde- oder gemeinschaftsbasierte Sorgemodelle, in den Blick nehmen.

Teil 4 greift diese Kernfrage erneut in einem separaten Schlusskapitel auf. Es wird darauf hingewiesen, dass mangelnde Standards auch ein Risiko für die zu Betreuenden darstellen. Geht es in diesem Zusammenhang hauptsächlich um (mangelnde) professionelle Standards, so ist doch auch klar, dass überforderte und übermüdete Betreuerinnen schwerlich gute Sorge leisten können. Die Frage bleibt, ob eine Rund-um-die-Uhr-Pflege im Privathaushalt in einer 1 : 1-Betreuung durch eine Person gewährleistet werden kann.

Die Autorinnen haben von vornherein ihren Forschungsgegenstand eingegrenzt: Es geht um agenturvermittelte Livein-Betreuung in Deutschland, der Schweiz und in Österreich. Das komplexe Geflecht zwischen Agenturen, Familien, zu Pflegenden und den Betreuungskräften wird von allen Facetten beleuchtet und faktenreich dargestellt.

Gleichwohl wird nicht ausreichend diskutiert, dass dieses Modell eines ist, was nicht flächendeckend zum Einsatz kommt und auch nicht kommen kann. Denn: Auch ausgebeutete, osteuropäische Live-in-Betreuerinnen müssen sich Familien erst einmal leisten können, auch wenn sie teilweise staatliche Unterstützungsleistungen geltend machen können. Schon aus diesem Grund müssen sich Gesellschaften fragen, wie sie zukünftig die Betreuung der zunehmenden Anzahl der Betagten organisieren wollen, sodass sowohl gute Pflege als auch gute Arbeit gewährleistet ist.

Das Buch ist für den wissenschaftlichen Diskurs geschrieben, verzichtet aber gleichzeitig auf einen bestimmten ,Jargon“, der sich nur denen erschließen würde, die im akade-

vergüten ist, ungeachtet dessen, dass ihr Arbeitsvertrag eine 30-Stunden-Woche vorsah, die mit EUR 950,-- netto monatlich vergütet wurde. 
mischen Milieu unterwegs sind. Es kann von daher allen Leser:innen empfohlen werden, die sich mit dem Thema beschäftigen wollen oder müssen. Es benennt Defizite, diskutiert erste Lösungen und regt zu weiterführenden Diskussionen und Überlegungen an. Ich würde mir wünschen, dass das Buch im akademischen Raum Forscher:innen motiviert zu überlegen, wie Modelle der guten Altenbetreuung aussehen können, die sich an den Bedürfnissen der zu Betreuenden orientieren und gute Arbeit einschließen.

Darüber hinaus braucht es einen breiten gesellschaftlichen Diskurs aller Beteiligten und den Willen der Politik, die Lücken zu schließen, die zu einem Geschäftsmodell geführt haben, das offensichtlich gegen die Vorgaben des ILOÜbereinkommens 189 verstößt (Gleichbehandlung mit anderen Arbeitnehmer:innen).
Dieser gesellschaftliche Diskurs, mit in der Folge politischem Handeln, kann aber nur dann erfolgreich sein, wenn die Betreuerinnen selbst beteiligt sind. „Nothing for us without us" ist das Motto der internationalen Föderation der Hausangestellten (IDWF ${ }^{2}$ : eine Voraussetzung für nachhaltige Veränderungen überall in der Welt und auch in Europa.

Haftungsausschluss. Publisher's note: Copernicus Publications remains neutral with regard to jurisdictional claims in published maps and institutional affiliations.

Interessenkonflikt. Die Autorin erklärt, dass kein Interessenkonflikt besteht.

\footnotetext{
${ }^{2}$ International Domestic Workers Federation (https://idwfed.org/ en, letzter Zugriff: 28. November 2021).
} 